\title{
Statistical equilibrium and photospheric abundance of silicon in the Sun and in Vega
}

\author{
S. Wedemeyer ${ }^{\star}$ \\ Institut für Theoretische Physik und Astrophysik, Universität Kiel, 24098 Kiel, Germany
}

Received 12 February 2001 / Accepted 2 May 2001

\begin{abstract}
Based on detailed non-LTE calculations, an updated determination of the abundance of silicon in the Sun and Vega is presented. The model atom includes neutral and singly ionized stages of silicon with 115 energy levels and 84 line transitions. Non-LTE effects are found to be quite small in the Sun. The mean non-LTE abundance correction is -0.010 dex with respect to standard LTE calculations, leading to a solar abundance of $\log \epsilon_{\mathrm{NLTE}}=7.550 \pm 0.056$. For the prototype A0 V star Vega the non-LTE effects are small, too. With a non-LTE abundance correction of $\Delta \log \epsilon=-0.054$, a silicon abundance of $\log \epsilon_{\mathrm{NLTE}}=6.951 \pm 0.100$ is derived, implying a deficiency of -0.599 dex with respect to the Sun. This confirms the classification of Vega as a mild $\lambda$ Boo star.
\end{abstract}

Key words. Sun: abundances - stars: abundances - atomic data

\section{Introduction}

For many astrophysical applications, an accurate knowledge of the silicon abundance is required. Silicon is not only an important reference element for comparing various types of cosmic matter (e.g. meteorites) with the Sun but also one of the main electron contributors (next to Fe and $\mathrm{Mg}$ ) and opacity sources in the near UV in the atmospheres of cool stars. Furthermore, the C/Si abundance ratio is an indicator of gas-dust separation in A stars with superficial abundance anomalies like $\lambda$ Boo stars (Stürenburg 1993).

The most widely used sources of solar (photospheric) abundances, the compilation by Anders \& Grevesse (1989) and its updates (e.g. Grevesse \& Sauval 1998), are based on standard abundance analyses employing 1D solar models and, in most cases, assuming LTE (local thermodynamic equilibrium). But for a accurate abundance determination, the simplifying assumption of LTE should be replaced by a detailed non-LTE study.

In the Sun, abundance deviations due to non-LTE effects are generally small, as can be seen from former calculations: +0.05 dex for Fe I (Steenbock 1985), -0.07 dex for C I (Stürenburg \& Holweger 1991) and -0.05 dex $(-0.01 \ldots-0.06$ dex $)$ for N I/II (Rentzsch-Holm 1996). Nevertheless, exact solar values are indispensable, as the Sun serves as a reference for investigations of other stars.

\footnotetext{
* Tables 7,8 and 9 are only available in electronic form at http://www. edpsciences.org

$\star \star$ e-mail: wedemeyer@astrophysik.uni-kiel.de
}

The A0V star Vega (HR 7001) is well studied in the context of abundance determination, and non-LTE calculations have been carried out for various elements (e.g. Gigas 1988; Takeda 1992). Its chemical composition shows a metal deficiency with respect to the Sun resembling the pattern of $\lambda$ Boo stars (Venn \& Lambert 1990; Holweger \& Rentzsch-Holm 1995). Therefore the former standard star Vega has turned into an important example of A stars with abundance anomalies.

For most elements, non-LTE corrections are small but not negligible, for example $-0.05 \operatorname{dex}(-0.16 \ldots 0.00 \mathrm{dex})$ for C I (Stürenburg \& Holweger 1990), -0.32 dex $(-0.16 \ldots-0.53$ dex $)$ for N I/II (Rentzsch-Holm 1996), $-1 \ldots-0.02$ dex for O I (Takeda 1992).

The presented calculations were carried out with the Kiel non-LTE code (Steenbock \& Holweger 1984) which uses the computational scheme developed by Auer \& Heasley (1976). Non-LTE calculations require various input data, such as a stellar atmosphere and a model atom which accounts for the relevant atomic properties. The resulting silicon abundances were derived with the program LINFOR, an updated and augmented Fortran version of the program by Baschek et al. (1966) devised by H. Holweger, M. Steffen and W. Steenbock at Kiel.

In Sect. 2 the atomic data used for the model atom are described. In Sects. 3 and 4 the non-LTE calculations and abundance determination are outlined for the Sun and for Vega, respectively. 
Table 1. Energy levels included in the model atom.

\begin{tabular}{|c|c|c|c|c|c|c|c|c|c|c|c|c|}
\hline \multicolumn{2}{|c|}{ no. } & config. & term & $E(\mathrm{eV})$ & $g_{i}$ & & no. & config. & term & $E(\mathrm{eV})$ & $g_{i}$ & \\
\hline$\overline{\mathrm{Si} \mathrm{I}}$ & 1 & $3 \mathrm{~s}^{2} 3 \mathrm{p}^{2}$ & ${ }^{3} \mathrm{P}$ & 0.0186 & 9 & & 59 & $3 \mathrm{~s}^{2} 3 \mathrm{p}\left({ }^{2} \mathrm{P}^{\mathrm{o}}\right) 5 \mathrm{f}$ & ${ }^{2}[9 / 2]$ & 7.6394 & 20 & \\
\hline & 2 & $3 s^{2} 3 p^{2}$ & ${ }^{1} \mathrm{D}$ & 0.7810 & 5 & & 60 & $3 \mathrm{~s}^{2} 3 \mathrm{p}\left({ }^{2} \mathrm{P}^{\mathrm{o}}\right) 5 \mathrm{~g}$ & ${ }^{2}[9 / 2]^{\mathrm{o}}$ & 7.6398 & 20 & * \\
\hline & 3 & $3 s^{2} 3 p^{2}$ & ${ }^{1} \mathrm{~S}$ & 1.9087 & 1 & & 61 & $3 \mathrm{~s}^{2} 3 \mathrm{p}\left({ }^{2} \mathrm{P}^{\mathrm{o}}\right) 5 \mathrm{~g}$ & ${ }^{2}[7 / 2]^{\circ}$ & 7.6411 & 16 & * \\
\hline & 4 & $3 \mathrm{~s} 3 \mathrm{p}^{3}\left({ }^{4} \mathrm{P}\right)$ & ${ }^{5} \mathrm{~S}^{\mathrm{O}}$ & 4.1319 & 5 & & 62 & $3 \mathrm{~s}^{2} 3 \mathrm{p}\left({ }^{2} \mathrm{P}^{\mathrm{o}}\right) 5 \mathrm{~g}$ & ${ }^{2}[11 / 2]^{\mathrm{o}}$ & 7.6429 & 24 & * \\
\hline & 5 & $3 \mathrm{~s}^{2} 3 \mathrm{p}\left({ }^{2} \mathrm{P}^{\mathrm{o}}\right) 4 \mathrm{~s}$ & ${ }^{3} \mathrm{P}^{\mathrm{o}}$ & 4.9420 & 9 & & 63 & $3 \mathrm{~s}^{2} 3 \mathrm{p}\left({ }^{2} \mathrm{P}^{\mathrm{o}}\right) 5 \mathrm{f}$ & $2[3 / 2]$ & 7.6434 & 8 & \\
\hline & 6 & $3 \mathrm{~s}^{2} 3 \mathrm{p}\left({ }^{2} \mathrm{P}^{\mathrm{o}}\right) 4 \mathrm{~s}$ & ${ }^{1} \mathrm{P}^{\mathrm{o}}$ & 5.0824 & 3 & & 64 & $3 \mathrm{~s}^{2} 3 \mathrm{p}\left({ }^{2} \mathrm{P}^{\mathrm{o}}\right) 5 \mathrm{~g}$ & ${ }^{2}[5 / 2]^{\mathrm{o}}$ & 7.6442 & 12 & * \\
\hline & 7 & $3 \mathrm{~s} 3 \mathrm{p}^{3}$ & ${ }^{3} \mathrm{D}^{\mathrm{o}}$ & 5.6169 & 15 & & 65 & $3 \mathrm{~s}^{2} 3 \mathrm{p}\left({ }^{2} \mathrm{P}^{\mathrm{o}}\right) 7 \mathrm{~s}$ & $(3 / 2,1 / 2)^{\mathrm{o}}$ & 7.6679 & 8 & \\
\hline & 8 & $3 \mathrm{~s}^{2} 3 \mathrm{p} 4 \mathrm{p}$ & ${ }^{1} \mathrm{P}$ & 5.8625 & 3 & & 66 & $3 \mathrm{~s}^{2} 3 \mathrm{p} 5 \mathrm{~d}$ & ${ }^{3} \mathrm{P}^{\mathrm{o}}$ & 7.6730 & 9 & * \\
\hline & 9 & $3 s^{2} 3 p 3 d$ & ${ }^{1} \mathrm{D}^{\mathrm{o}}$ & 5.8708 & 5 & & 67 & $3 \mathrm{~s}^{2} 3 \mathrm{p} 6 \mathrm{~d}$ & ${ }^{1} \mathrm{D}^{\mathrm{o}}$ & 7.7065 & 5 & \\
\hline & 10 & $3 s^{2} 3 p 4 p$ & ${ }^{3} \mathrm{D}$ & 5.9713 & 15 & & 68 & $3 \mathrm{~s}^{2} 3 \mathrm{p}\left({ }^{2} \mathrm{P}^{\mathrm{o}}\right) 7 \mathrm{p}$ & $(1 / 2,1 / 2)$ & 7.7101 & 4 & \\
\hline & 11 & $3 \mathrm{~s}^{2} 3 \mathrm{p} 4 \mathrm{p}$ & ${ }^{3} \mathrm{P}$ & 6.0911 & 9 & & 69 & $3 \mathrm{~s}^{2} 3 \mathrm{p}\left({ }^{2} \mathrm{P}^{\mathrm{o}}\right) 7 \mathrm{p}$ & $(1 / 2,3 / 2)$ & 7.7156 & 8 & \\
\hline & 12 & $3 s^{2} 3 p 4 p$ & ${ }^{3} \mathrm{~S}$ & 6.1248 & 3 & & 70 & $3 s^{2} 3 p 6 d$ & ${ }^{3} \mathrm{~F}^{\mathrm{o}}$ & 7.7414 & 21 & \\
\hline & 13 & $3 \mathrm{~s}^{2} 3 \mathrm{p} 3 \mathrm{~d}$ & ${ }^{3} \mathrm{~F}^{\mathrm{o}}$ & 6.1959 & 21 & & 71 & $3 \mathrm{~s}^{2} 3 \mathrm{p}\left({ }^{2} \mathrm{P}^{\mathrm{o}}\right) 7 \mathrm{p}$ & $(3 / 2,3 / 2)$ & 7.7491 & 16 & \\
\hline & 14 & $3 s^{2} 3 p 4 p$ & ${ }^{1} \mathrm{D}$ & 6.2227 & 5 & & 72 & $3 \mathrm{~s}^{2} 3 \mathrm{p}\left({ }^{2} \mathrm{P}^{\mathrm{o}}\right) 7 \mathrm{p}$ & $(3 / 2,1 / 2)$ & 7.7527 & 8 & \\
\hline & 15 & $3 \mathrm{~s}^{2} 3 \mathrm{p} 3 \mathrm{~d}$ & ${ }^{3} \mathrm{P}^{\mathrm{o}}$ & 6.2653 & 9 & & 73 & $3 \mathrm{~s}^{2} 3 \mathrm{p} 6 \mathrm{~d}$ & ${ }^{1} \mathrm{P}^{\mathrm{o}}$ & 7.7697 & 3 & \\
\hline & 16 & $3 \mathrm{~s}^{2} 3 \mathrm{p} 4 \mathrm{p}$ & ${ }^{1} \mathrm{~S}$ & 6.3990 & 1 & & 74 & $3 \mathrm{~s}^{2} 3 \mathrm{p}\left({ }^{2} \mathrm{P}^{\mathrm{o}}\right) 6 \mathrm{f}$ & ${ }^{2}[7 / 2]$ & 7.7700 & 16 & \\
\hline & 17 & $3 \mathrm{~s}^{2} 3 \mathrm{p} 3 \mathrm{~d}$ & ${ }^{1} \mathrm{~F}^{\mathrm{o}}$ & 6.6161 & 7 & & 75 & $3 \mathrm{~s}^{2} 3 \mathrm{p}\left({ }^{2} \mathrm{P}^{\mathrm{o}}\right) 6 \mathrm{f}$ & $2[5 / 2]$ & 7.7701 & 12 & \\
\hline & 18 & $3 \mathrm{~s}^{2} 3 \mathrm{p} 3 \mathrm{~d}$ & ${ }^{1} \mathrm{P}^{\mathrm{o}}$ & 6.6192 & 3 & & & & & & & \\
\hline & 19 & $3 s^{2} 3 p 3 d$ & ${ }^{3} \mathrm{D}^{\circ}$ & 6.7232 & 15 & Si II & 76 & $3 \mathrm{~s}^{2}\left({ }^{1} \mathrm{~S}\right) 3 \mathrm{p}$ & ${ }^{2} \mathrm{P}^{\mathrm{o}}$ & 0.0237 & 6 & \\
\hline & 20 & $3 s^{2} 3 p 5 s$ & ${ }^{3} \mathrm{P}^{\mathrm{o}}$ & 6.7478 & 9 & & 77 & $3 \mathrm{~s} 3 \mathrm{p}^{2}$ & ${ }^{4} \mathrm{P}$ & 5.3316 & 12 & * \\
\hline & 21 & $3 s^{2} 3 p 5 s$ & ${ }^{1} \mathrm{P}^{\mathrm{o}}$ & 6.8031 & 3 & & 78 & $3 \mathrm{~s} 3 \mathrm{p}^{2}$ & ${ }^{2} \mathrm{D}$ & 6.8587 & 10 & \\
\hline & 22 & $3 \mathrm{~s}^{2} 3 \mathrm{p} 4 \mathrm{~d}$ & ${ }^{1} \mathrm{D}^{\circ}$ & 7.0055 & 5 & & 79 & $3 s^{2}\left({ }^{1} s\right) 4 s$ & ${ }^{2} \mathrm{~S}$ & 8.1210 & 2 & \\
\hline & 23 & $3 \mathrm{~s}^{2} 3 \mathrm{p} 4 \mathrm{~d}$ & ${ }^{3} \mathrm{P}^{\mathrm{o}}$ & 7.0298 & 9 & & 80 & $3 \mathrm{~s} 3 \mathrm{p}^{2}$ & ${ }^{2} \mathrm{~S}$ & 9.5054 & 2 & \\
\hline & 24 & $3 s^{2} 3 p 5 p$ & ${ }^{1} \mathrm{P}$ & 7.0399 & 3 & & 81 & $3 \mathrm{~s}^{2}\left({ }^{1} \mathrm{~S}\right) 3 \mathrm{~d}$ & ${ }^{2} \mathrm{D}$ & 9.8380 & 10 & \\
\hline & 25 & $3 \mathrm{~s}^{2} 3 \mathrm{p} 5 \mathrm{p}$ & ${ }^{3} \mathrm{D}$ & 7.0787 & 15 & & 82 & $3 \mathrm{~s}^{2}\left({ }^{1} \mathrm{~S}\right) 4 \mathrm{p}$ & ${ }^{2} \mathrm{P}^{\mathrm{o}}$ & 10.0715 & 6 & \\
\hline & 26 & $3 s^{2} 3 p 5 p$ & ${ }^{3} \mathrm{P}$ & 7.1170 & 9 & & 83 & $3 \mathrm{~s} 3 \mathrm{p}^{2}$ & ${ }^{2} \mathrm{P}$ & 10.4069 & 6 & \\
\hline & 27 & $3 \mathrm{~s}^{2} 3 \mathrm{p} 4 \mathrm{~d}$ & ${ }^{3} \mathrm{~F}^{\mathrm{o}}$ & 7.1277 & 21 & & 84 & $3 \mathrm{~s}^{2}\left({ }^{1} \mathrm{~s}\right) 5 \mathrm{~s}$ & ${ }^{2} \mathrm{~S}$ & 12.1471 & 2 & \\
\hline & 28 & $3 s^{2} 3 p 5 p$ & ${ }^{3} \mathrm{~S}$ & 7.1343 & 3 & & 85 & $3 \mathrm{~s}^{2}\left({ }^{1} \mathrm{~S}\right) 4 \mathrm{~d}$ & ${ }^{2} \mathrm{D}$ & 12.5255 & 10 & \\
\hline & 29 & $3 s^{2} 3 p 5 p$ & ${ }^{1} \mathrm{D}$ & 7.1660 & 5 & & 86 & $3 \mathrm{~s}^{2}\left({ }^{1} \mathrm{~S}\right) 4 \mathrm{f}$ & ${ }^{2} \mathrm{~F}^{\mathrm{O}}$ & 12.8394 & 14 & \\
\hline & 30 & $3 s^{2} 3 p 5 p$ & ${ }^{1} \mathrm{~S}$ & 7.2297 & 1 & & 87 & $3 s^{2}\left({ }^{1} S\right) 5 p$ & ${ }^{2} \mathrm{P}^{\mathrm{o}}$ & 12.8792 & 6 & \\
\hline & 31 & $3 \mathrm{~s}^{2} 3 \mathrm{p}\left({ }^{2} \mathrm{P}^{\mathrm{o}}\right) 4 \mathrm{f}$ & ${ }^{2}[5 / 2]$ & 7.2872 & 12 & & 88 & $3 \mathrm{~s} 3 \mathrm{p}\left({ }^{3} \mathrm{P}^{\mathrm{o}}\right) 3 \mathrm{~d}$ & ${ }^{2} \mathrm{D}^{\mathrm{o}}$ & 13.4901 & 10 & \\
\hline & 32 & $3 \mathrm{~s}^{2} 3 \mathrm{p}\left({ }^{2} \mathrm{P}^{\mathrm{o}}\right) 4 \mathrm{f}$ & ${ }^{3} \mathrm{~F}$ & 7.2888 & 16 & & 89 & $3 s^{2}\left({ }^{1} S\right) 6 s$ & ${ }^{2} \mathrm{~S}$ & 13.7852 & 2 & \\
\hline & 33 & $3 \mathrm{~s}^{2} 3 \mathrm{p} 4 \mathrm{~d}$ & ${ }^{1} \mathrm{P}^{\mathrm{o}}$ & 7.2905 & 3 & & 90 & $3 \mathrm{~s}^{2}\left({ }^{1} \mathrm{~S}\right) 5 \mathrm{~d}$ & ${ }^{2} \mathrm{D}$ & 13.9353 & 10 & \\
\hline & 34 & $3 \mathrm{~s}^{2} 3 \mathrm{p} 4 \mathrm{~d}$ & ${ }^{1} \mathrm{~F}^{\mathrm{o}}$ & 7.3019 & 7 & & 91 & $3 s^{2}\left({ }^{1} S\right) 5 f$ & ${ }^{2} \mathrm{~F}^{\mathrm{O}}$ & 14.1046 & 14 & \\
\hline & 35 & $3 \mathrm{~s}^{2} 3 \mathrm{p}\left({ }^{2} \mathrm{P}^{\mathrm{o}}\right) 4 \mathrm{f}$ & ${ }^{3} \mathrm{G}$ & 7.3196 & 16 & & 92 & $3 \mathrm{~s}^{2}\left({ }^{1} \mathrm{~S}\right) 6 \mathrm{p}$ & ${ }^{2} \mathrm{P}^{\mathrm{o}}$ & 14.1308 & 6 & \\
\hline & 36 & $3 \mathrm{~s}^{2} 3 \mathrm{p} 4 \mathrm{~d}$ & ${ }^{3} \mathrm{D}^{\mathrm{o}}$ & 7.3247 & 15 & & 93 & $3 \mathrm{~s}^{2}\left({ }^{1} \mathrm{~S}\right) 5 \mathrm{~g}$ & ${ }^{2} \mathrm{G}$ & 14.1563 & 18 & \\
\hline & 37 & $3 \mathrm{~s}^{2} 3 \mathrm{p}\left({ }^{2} \mathrm{P}^{\mathrm{o}}\right) 4 \mathrm{f}$ & ${ }^{2}[5 / 2]$ & 7.3288 & 12 & & 94 & $3 \mathrm{~s} 3 \mathrm{p}\left({ }^{3} \mathrm{P}^{\mathrm{o}}\right) 3 \mathrm{~d}$ & ${ }^{4} \mathrm{~F}^{\mathrm{O}}$ & 14.1858 & 28 & * \\
\hline & 38 & $3 \mathrm{~s}^{2} 3 \mathrm{p}\left({ }^{2} \mathrm{P}^{\mathrm{o}}\right) 4 \mathrm{f}$ & $2[9 / 2]$ & 7.3312 & 20 & & 95 & $3 \mathrm{~s} 3 \mathrm{p}\left({ }^{3} \mathrm{P}^{\mathrm{o}}\right) 4 \mathrm{~s}$ & ${ }^{4} \mathrm{P}^{\mathrm{o}}$ & 14.5136 & 12 & * \\
\hline & 39 & $3 \mathrm{~s}^{2} 3 \mathrm{p}\left({ }^{2} \mathrm{P}^{\mathrm{o}}\right) 4 \mathrm{f}$ & $2[3 / 2]$ & 7.3388 & 8 & & 96 & $3 \mathrm{~s}^{2}\left({ }^{1} \mathrm{~S}\right) 7 \mathrm{~s}$ & ${ }^{2} \mathrm{~S}$ & 14.6196 & 2 & \\
\hline & 40 & $3 \mathrm{~s}^{2} 3 \mathrm{p}\left({ }^{2} \mathrm{P}^{\mathrm{o}}\right) 6 \mathrm{~s}$ & ${ }^{3} \mathrm{P}^{\mathrm{o}}$ & 7.3474 & 4 & & 97 & $3 \mathrm{~s}^{2}\left({ }^{1} \mathrm{~S}\right) 6 \mathrm{~d}$ & ${ }^{2} \mathrm{D}$ & 14.6951 & 10 & \\
\hline & 41 & $3 \mathrm{~s}^{2} 3 \mathrm{p}\left({ }^{2} \mathrm{P}^{\mathrm{o}}\right) 6 \mathrm{~s}$ & $(3 / 2,1 / 2)^{\circ}$ & 7.3840 & 8 & & 98 & $3 \mathrm{~s}^{2}\left({ }^{1} \mathrm{~S}\right) 7 \mathrm{p}$ & ${ }^{2} \mathrm{P}^{\mathrm{o}}$ & 14.7870 & 6 & \\
\hline & 42 & $3 \mathrm{~s}^{2} 3 \mathrm{p}$ nd & ${ }^{3} \mathrm{P}^{\mathrm{o}}$ & 7.4344 & 9 & & 99 & $3 s^{2}\left({ }^{1} S\right) 6 f$ & ${ }^{2} \mathrm{~F}^{\mathrm{o}}$ & 14.7928 & 14 & \\
\hline & 43 & $3 \mathrm{~s}^{2} 3 \mathrm{p} 5 \mathrm{~d}$ & ${ }^{1} \mathrm{D}^{\mathrm{o}}$ & 7.4764 & 5 & & 100 & $3 \mathrm{~s}^{2}\left({ }^{1} \mathrm{~S}\right) 6 \mathrm{~g}$ & ${ }^{2} \mathrm{G}$ & 14.8258 & 18 & \\
\hline & 44 & $3 \mathrm{~s}^{2} 3 \mathrm{p}\left({ }^{2} \mathrm{P}^{\mathrm{o}}\right) 6 \mathrm{p}$ & $(1 / 2,1 / 2)$ & 7.4938 & 4 & & 101 & $3 \mathrm{~s} 3 \mathrm{p}\left({ }^{3} \mathrm{P}^{\mathrm{o}}\right) 4 \mathrm{~s}$ & ${ }^{2} \mathrm{P}^{\mathrm{o}}$ & 15.0693 & 6 & \\
\hline & 45 & $3 \mathrm{~s}^{2} 3 \mathrm{p}\left({ }^{2} \mathrm{P}^{\mathrm{o}}\right) 6 \mathrm{p}$ & $(1 / 2,3 / 2)$ & 7.5002 & 8 & & 102 & $3 s^{2}\left({ }^{1} S\right) 8 s$ & ${ }^{2} \mathrm{~S}$ & 15.1031 & 2 & \\
\hline & 46 & $3 \mathrm{~s}^{2} 3 \mathrm{p} 5 \mathrm{~d}$ & ${ }^{3} \mathrm{~F}^{\mathrm{O}}$ & 7.5324 & 21 & & 103 & $3 \mathrm{~s}^{2}\left({ }^{1} \mathrm{~S}\right) 7 \mathrm{~d}$ & ${ }^{2} \mathrm{D}$ & 15.1463 & 10 & \\
\hline & 47 & $3 \mathrm{~s}^{2} 3 \mathrm{p}\left({ }^{2} \mathrm{P}^{\mathrm{o}}\right) 6 \mathrm{p}$ & $(3 / 2,3 / 2)$ & 7.5403 & 16 & & 104 & $3 \mathrm{~s}^{2}\left({ }^{1} \mathrm{~S}\right) 7 \mathrm{f}$ & ${ }^{2} \mathrm{~F}^{\mathrm{O}}$ & 15.2073 & 14 & \\
\hline & 48 & $3 \mathrm{~s}^{2} 3 \mathrm{p}\left({ }^{2} \mathrm{P}^{\mathrm{o}}\right) 6 \mathrm{p}$ & $(3 / 2,1 / 2)$ & 7.5422 & 8 & & 105 & $3 \mathrm{~s}^{2}\left({ }^{1} \mathrm{~S}\right) 7 \mathrm{~g}$ & ${ }^{2} \mathrm{G}$ & 15.2296 & 18 & \\
\hline & 49 & $3 \mathrm{~s}^{2} 3 \mathrm{p}\left({ }^{2} \mathrm{P}^{\mathrm{o}}\right) 5 \mathrm{f}$ & ${ }^{2}[5 / 2]$ & 7.6008 & 12 & & 106 & $3 p^{3}$ & ${ }^{4} \mathrm{~S}^{\mathrm{O}}$ & 15.2542 & 4 & * \\
\hline & 50 & $3 \mathrm{~s}^{2} 3 \mathrm{p}\left({ }^{2} \mathrm{P}^{\mathrm{o}}\right) 5 \mathrm{f}$ & ${ }^{3} \mathrm{~F}$ & 7.6010 & 16 & & 107 & $3 \mathrm{~s}^{2}\left({ }^{1} \mathrm{~S}\right) 8 \mathrm{p}$ & ${ }^{2} \mathrm{P}^{\mathrm{o}}$ & 15.2656 & 6 & \\
\hline & 51 & $3 \mathrm{~s}^{2} 3 \mathrm{p} 5 \mathrm{~d}$ & ${ }^{1} \mathrm{P}^{\mathrm{o}}$ & 7.6011 & 3 & & 108 & $3 s^{2}\left({ }^{1} s\right) 9 s$ & ${ }^{2} \mathrm{~S}$ & 15.4084 & 2 & \\
\hline & 52 & $3 \mathrm{~s}^{2} 3 \mathrm{p}\left({ }^{2} \mathrm{P}^{\mathrm{o}}\right) 5 \mathrm{~g}$ & ${ }^{2}[7 / 2]^{\circ}$ & 7.6060 & 16 & & 109 & $3 \mathrm{~s} 3 \mathrm{p}\left({ }^{3} \mathrm{P}^{\mathrm{o}}\right) 3 \mathrm{~d}$ & ${ }^{4} \mathrm{D}^{\mathrm{o}}$ & 15.4204 & 20 & * \\
\hline & 53 & $3 \mathrm{~s}^{2} 3 \mathrm{p}\left({ }^{2} \mathrm{P}^{\mathrm{o}}\right) 5 \mathrm{~g}$ & $2^{2}[9 / 2]^{\mathrm{o}}$ & 7.6061 & 20 & & 110 & $3 \mathrm{~s}^{2}\left({ }^{1} \mathrm{~S}\right) 8 \mathrm{~d}$ & ${ }^{2} \mathrm{D}$ & 15.4355 & 10 & \\
\hline & 54 & $3 s^{2} 3 p 5 d$ & ${ }^{1} \mathrm{~F}^{\mathrm{o}}$ & 7.6156 & 7 & & 111 & $3 \mathrm{~s} 3 \mathrm{p}\left({ }^{3} \mathrm{P}^{\mathrm{o}}\right) 3 \mathrm{~d}$ & ${ }^{4} \mathrm{P}^{\mathrm{o}}$ & 15.4479 & 12 & * \\
\hline & 55 & $3 \mathrm{~s}^{2} 3 \mathrm{p} 5 \mathrm{~d}$ & ${ }^{3} \mathrm{D}^{\circ}$ & 7.6276 & 15 & & 112 & $3 \mathrm{~s}^{2}\left({ }^{1} \mathrm{~S}\right) 8 \mathrm{f}$ & ${ }^{2} \mathrm{~F}^{\mathrm{O}}$ & 15.4760 & 14 & \\
\hline & 56 & $3 \mathrm{~s}^{2} 3 \mathrm{p}\left({ }^{2} \mathrm{P}^{\mathrm{o}}\right) 5 \mathrm{f}$ & ${ }^{3} \mathrm{G}$ & 7.6329 & 16 & & 113 & $3 \mathrm{~s}^{2}\left({ }^{1} \mathrm{~S}\right) 8 \mathrm{~g}$ & ${ }^{2} \mathrm{G}$ & 15.4916 & 18 & \\
\hline & 57 & $3 \mathrm{~s}^{2} 3 \mathrm{p}\left({ }^{2} \mathrm{P}^{\mathrm{o}}\right) 7 \mathrm{~s}$ & ${ }^{3} \mathrm{P}^{\mathrm{o}}$ & 7.6351 & 4 & & 114 & $3 \mathrm{~s}^{2}\left({ }^{1} \mathrm{~S}\right) 9 \mathrm{p}$ & ${ }^{2} \mathrm{P}^{\mathrm{o}}$ & 15.5018 & 6 & \\
\hline & 58 & $3 \mathrm{~s}^{2} 3 \mathrm{p}\left({ }^{2} \mathrm{P}^{\mathrm{o}}\right) 5 \mathrm{f}$ & ${ }^{3} \mathrm{D}$ & 7.6372 & 12 & & 115 & $3 \mathrm{~s} 3 \mathrm{p}\left({ }^{3} \mathrm{P}^{\mathrm{o}}\right) 3 \mathrm{~d}$ & ${ }^{2} \mathrm{P}^{\mathrm{o}}$ & 15.6531 & 6 & \\
\hline
\end{tabular}


Table 2. Line transitions used in the model atom.

\begin{tabular}{|c|c|c|c|c|c|c|c|c|c|c|c|c|c|}
\hline no. & mult. & $i$ & $k$ & $\lambda(\AA)$ & $\log g f$ & & no. & mult. & $i$ & $k$ & $\lambda(\AA)$ & $\log g f$ & \\
\hline Si I-1 & $0.01 \mathrm{~F}$ & 1 & 2 & 16263.251 & -9.126 & $\mathrm{~F}$ & Si I-43 & 31 & 8 & 22 & 10846.829 & +0.150 & $\mathrm{~F}$ \\
\hline Si I-2 & $1 \mathrm{~F}$ & 1 & 3 & 6567.708 & -9.634 & $\mathrm{~F}$ & Si I-44 & 32.02 & 8 & 33 & 8680.080 & -3.191 & $\mathrm{~F}$ \\
\hline Si I-3 & 0.01 & 1 & 4 & 3014.243 & -4.522 & $\mathrm{~F}$ & Si I-45 & 34 & 8 & 41 & 8093.231 & -1.354 & $\mathrm{~F}$ \\
\hline Si I-4 & UV 1 & 1 & 5 & 2517.485 & +0.173 & $\mathrm{~F}$ & Si I-46 & 36 & 8 & 43 & 7680.265 & -0.691 & $\mathrm{~F}$ \\
\hline Si I-5 & UV 2 & 1 & 6 & 2448.510 & -2.264 & $\mathrm{~F}$ & Si I-47 & 38 & 8 & 67 & 6721.840 & -0.938 & $\mathrm{~F}$ \\
\hline Si I-6 & UV 3 & 1 & 7 & 2213.972 & -0.217 & $\mathrm{~F}$ & Si I-48 & 42.21 & 10 & 20 & 15964.678 & +0.456 & $\mathrm{~F}$ \\
\hline Si I-7 & UV 7 & 1 & 15 & 1984.967 & -0.314 & $\mathrm{~V}$ & Si I-49 & 53 & 10 & 27 & 10720.959 & +0.672 & $\mathrm{~F}$ \\
\hline Si I-8 & UV 8 & 1 & 17 & 1881.854 & -1.922 & $\mathrm{~V}$ & Si I-50 & 57 & 10 & 46 & 7939.870 & +0.061 & $\mathrm{~F}$ \\
\hline Si I-9 & UV 10 & 1 & 19 & 1849.336 & +0.387 & $\mathrm{~V}$ & Si I-51 & 60 & 10 & 70 & 7002.660 & -0.380 & $\mathrm{~F}$ \\
\hline Si I-10 & UV 11 & 1 & 20 & 1842.489 & -0.663 & $\mathrm{~V}$ & Si I-52 & & 11 & 21 & 17205.800 & -1.450 & $\mathrm{~L}$ \\
\hline Si I-11 & $2 \mathrm{~F}$ & 2 & 3 & 10991.400 & -7.839 & $\mathrm{~F}$ & Si I-53 & & 12 & 20 & 20343.900 & -0.810 & $\mathrm{~L}$ \\
\hline Si I-12 & 1 & 2 & 5 & 2979.674 & -2.045 & $\mathrm{~F}$ & Si II-1 & UV 0.01 & 76 & 77 & 2325.848 & -4.193 & $\mathrm{~F}$ \\
\hline Si I-13 & UV 43 & 2 & 6 & 2881.577 & -0.151 & $\mathrm{~F}$ & Si II-2 & UV 1 & 76 & 78 & 1813.980 & -1.474 & $\mathrm{~F}$ \\
\hline Si I-14 & UV 45 & 2 & 9 & 2435.154 & -0.680 & $\mathrm{~V}$ & Si II-3 & UV 2 & 76 & 79 & 1531.183 & -0.108 & $\mathrm{~F}$ \\
\hline Si I-15 & UV 48 & 2 & 17 & 2124.111 & +0.533 & $\mathrm{~V}$ & Si II-4 & UV 3 & 76 & 80 & 1307.636 & -0.249 & $\mathrm{~F}$ \\
\hline Si I-16 & UV 49 & 2 & 18 & 2122.990 & -0.915 & $\mathrm{~V}$ & Si II-5 & UV 4 & 76 & 81 & 1263.313 & +0.759 & $\mathrm{~F}$ \\
\hline Si I-17 & UV 50 & 2 & 19 & 2084.462 & -1.573 & $\mathrm{~V}$ & Si II-6 & UV 5 & 76 & 83 & 1194.096 & +0.742 & $\mathrm{~F}$ \\
\hline Si I-18 & UV 51 & 2 & 20 & 2082.021 & -2.229 & $\mathrm{~V}$ & Si II-7 & UV 5.01 & 76 & 84 & 1022.698 & -0.902 & $\mathrm{~F}$ \\
\hline Si I-19 & UV 52 & 2 & 21 & 2058.133 & -1.030 & $\mathrm{~V}$ & Si II-8 & UV 6 & 76 & 85 & 991.745 & +0.072 & $\mathrm{~F}$ \\
\hline Si I-20 & UV 53 & 2 & 22 & 1991.853 & -2.189 & $\mathrm{~V}$ & Si II-9 & & 77 & 82 & 2618.212 & -4.135 & $\mathrm{~F}$ \\
\hline Si I-21 & 2 & 3 & 5 & 4102.935 & -2.916 & $\mathrm{~F}$ & Si II-10 & & 77 & 86 & 1652.411 & -3.759 & $\mathrm{~F}$ \\
\hline Si I-22 & 3 & 3 & 6 & 3905.521 & -1.092 & $\mathrm{~F}$ & Si II-11 & & 77 & 106 & 1249.510 & +0.551 & $\mathrm{~F}$ \\
\hline Si I-23 & UV 82 & 3 & 15 & 2842.333 & -3.274 & $\mathrm{~V}$ & Si II-12 & 1 & 78 & 82 & 3858.050 & -0.426 & $\mathrm{~F}$ \\
\hline Si I-24 & UV 83 & 3 & 18 & 2631.282 & -0.520 & $\mathrm{~V}$ & Si II-13 & UV 9 & 78 & 86 & 2072.430 & -0.045 & $\mathrm{~F}$ \\
\hline Si I-25 & UV 86 & 3 & 21 & 2532.381 & -1.200 & $\mathrm{~V}$ & Si II-14 & 2 & 79 & 82 & 6355.200 & +0.406 & $\mathrm{~F}$ \\
\hline Si I-26 & 4 & 5 & 10 & 12045.959 & +0.744 & $\mathrm{~F}$ & Si II-15 & 3 & 81 & 86 & 4129.760 & +0.706 & $\mathrm{~F}$ \\
\hline Si I-27 & 5 & 5 & 11 & 10789.570 & +0.539 & $\mathrm{~F}$ & Si II-16 & UV 17 & 81 & 91 & 2905.130 & +0.100 & $\mathrm{~F}$ \\
\hline Si I-28 & 6 & 5 & 12 & 10482.452 & +0.074 & $\mathrm{~F}$ & Si II-17 & UV 18 & 81 & 99 & 2501.570 & -0.269 & $\mathrm{~F}$ \\
\hline Si I-29 & & 5 & 14 & 9768.400 & -2.300 & $\mathrm{~L}$ & Si II-18 & 4 & 82 & 84 & 5971.800 & +0.109 & $\mathrm{~F}$ \\
\hline Si I-30 & 9 & 5 & 25 & 5800.880 & -0.866 & $\mathrm{~F}$ & Si II-19 & 5 & 82 & 85 & 5051.010 & +0.662 & $\mathrm{~F}$ \\
\hline Si I-31 & 10 & 5 & 26 & 5698.800 & -0.867 & $\mathrm{~F}$ & Si II-20 & 6 & 82 & 89 & 3337.590 & -0.823 & $\mathrm{~F}$ \\
\hline Si I-32 & 11 & 5 & 28 & 5653.900 & -1.170 & $\mathrm{~F}$ & Si II-21 & 7 & 82 & 90 & 3207.970 & -0.149 & $\mathrm{~F}$ \\
\hline Si I-33 & 11.04 & 5 & 45 & 4846.646 & -1.527 & $\mathrm{~F}$ & Si II-22 & UV 19 & 82 & 96 & 2725.220 & -1.272 & $\mathrm{~F}$ \\
\hline Si I-34 & 11.06 & 5 & 48 & 4768.462 & -1.155 & $\mathrm{~F}$ & Si II-23 & & 83 & 86 & 5113.168 & -3.514 & $\mathrm{~F}$ \\
\hline Si I-35 & 11.12 & 6 & 8 & 15892.767 & -0.036 & $\mathrm{~F}$ & Si II-24 & 7.02 & 85 & 91 & 7849.400 & +0.735 & $\mathrm{~F}$ \\
\hline Si I-36 & & 6 & 11 & 12390.200 & -1.710 & $\mathrm{~L}$ & Si II-25 & 7.03 & 85 & 99 & 5466.720 & +0.213 & $\mathrm{~F}$ \\
\hline Si I-37 & & 6 & 12 & 11890.500 & -2.090 & $\mathrm{~L}$ & Si II-26 & 7.05 & 85 & 104 & 4621.600 & -0.144 & $\mathrm{~F}$ \\
\hline Si I-38 & 13 & 6 & 14 & 10872.520 & +0.309 & $\mathrm{~F}$ & Si II-27 & 7.12 & 86 & 97 & 6679.650 & -1.028 & $\mathrm{~F}$ \\
\hline Si I-39 & 14 & 6 & 16 & 9413.506 & -0.445 & $\mathrm{~F}$ & Si II-28 & 7.19 & 87 & 96 & 7121.700 & -0.642 & $\mathrm{~F}$ \\
\hline Si I-40 & 14.01 & 6 & 24 & 6331.957 & -3.744 & $\mathrm{~F}$ & Si II-29 & 7.20 & 87 & 97 & 6826.000 & -0.042 & $\mathrm{~F}$ \\
\hline Si I-41 & 16 & 6 & 29 & 5948.540 & -1.234 & $\mathrm{~F}$ & Si II-30 & 7.21 & 87 & 102 & 5573.430 & -1.096 & $\mathrm{~F}$ \\
\hline Si I-42 & 17 & 6 & 30 & 5772.145 & -1.745 & $\mathrm{~F}$ & Si II-31 & 7.23 & 87 & 108 & 4900.700 & -1.418 & $\mathrm{~F}$ \\
\hline
\end{tabular}

$\mathrm{F}=$ Fuhr \& Wiese (1998), L = Lambert \& Luck (1978), V = VALD (Piskunov et al. 1995 ; Kurucz 1993).

\section{Atomic data}

The silicon model atom accounts for the most important levels and transitions of Si I and Si II and comprises 115 energy levels and 84 line transitions. For Si I (ionization limit at $8.15 \mathrm{eV}) 75$ energy levels up to $7.77 \mathrm{eV}$ and 53 line transitions were included, for Si II (ionization limit at $16.35 \mathrm{eV}$ ) 40 energy levels up to $15.65 \mathrm{eV}$ and 31 line transitions were taken into account. The atomic data are listed in Tables 1 and 2, while Fig. 1 shows the corresponding Grotrian diagrams.
The data for the energy levels were adopted from a compilation of Martin \& Zalubas (1983) which is available from the internet server of the National Institute of Standards and Technology (NIST, http://physics.nist.gov). This source also includes some unpublished measurements. It should be mentioned that apart from LS coupling, other schemes $\left(J l\left(j_{\mathrm{c}}[K]_{J}^{\pi}\right)\right.$, $J j\left((j, J)^{\pi}\right)$ were found for Si I. For consistency and practical reasons the concerned energy levels were designated to LS coupling if possible (Table 1).

Data for the line transitions used in the model atom (Table 2) were obtained from the NIST server, the 
${ }^{1} S^{1} P^{1} P^{01} D^{1} D^{01} F^{03} S^{3} P^{3} P^{03} D^{3} D^{03} F^{3} F^{\circ 3} G^{5} S^{0} \mathrm{Jj}$ J

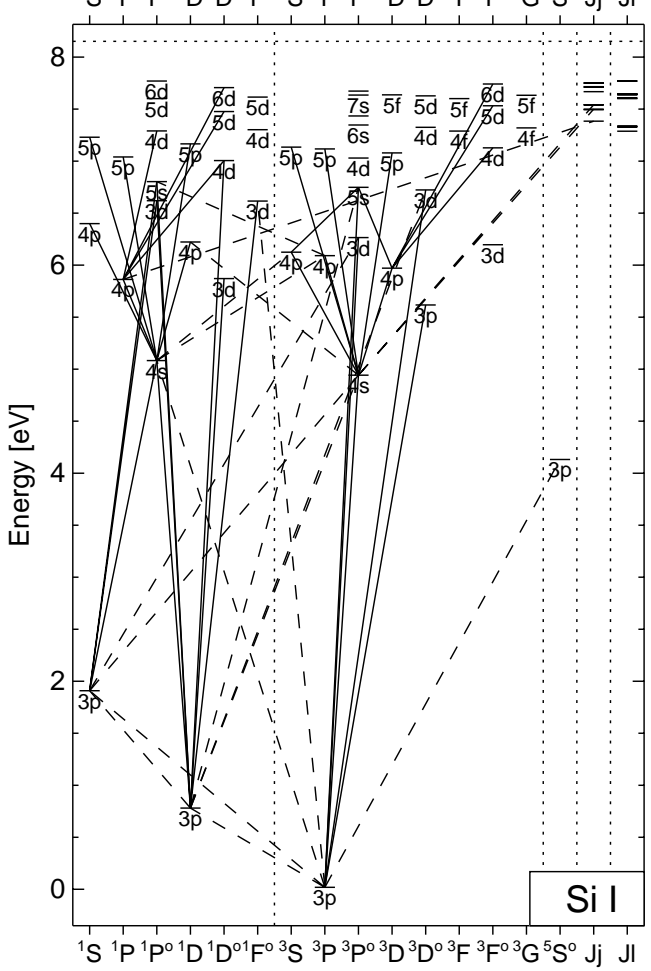

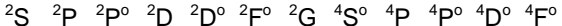

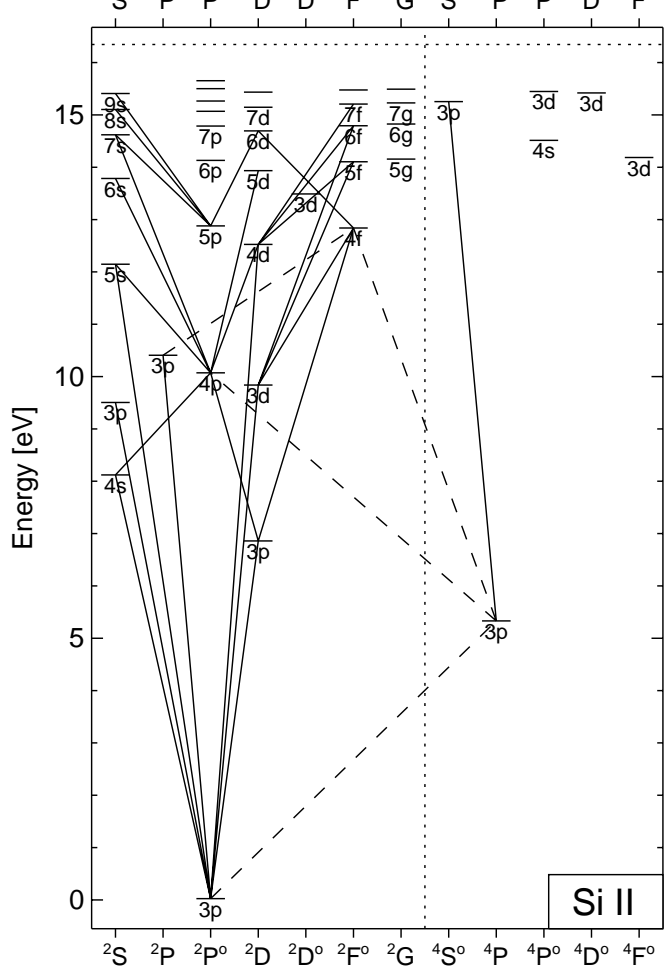

Fig. 1. Grotrian diagrams of the silicon model atom including Si I with 75 energy levels and 53 line transitions and Si II with 40 levels and 31 transitions.

Vienna Atomic Line Data Base (Piskunov et al. 1995; Kurucz 1993) and Lambert \& Luck (1978). The NIST data refer to the compilation of Wiese et al. (1969) and the newer version by Fuhr \& Wiese (1998). The latter provides improved transition probabilities for some line transitions.

Photoionization cross-sections were taken from the Opacity Project (Seaton et al. 1992, 1994) for almost all energy levels with the exception of eleven mostly high excited ones (marked with ${ }^{*}$ in Table 1 ). In these cases the Kramers Gaunt aproximation for hydrogen-like atoms as given by Allen (1973) was used.

The majority of the more important electron collisional cross-sections of Si I were calculated using the tables given by Sobelman et al. (1981). Transistions not covered by the Sobelman tables were treated with the formulas compiled by Drawin (1967) but additionally needed to be scaled to the corresponding maximum crosssections. For optically allowed transitions, the maximum cross-sections were calculated with the approximation of Van Regemorter (1962) using all available oscillator strengths and additional values from the Opacity Project. In all other cases, especially for optically forbidden transitions, the cross-sections were scaled with the collision strength formula described by Allen (1973).

Drawin (1967) also provides an estimate for collisional ionization by electrons, which was applied here, and for inelastic collisions with neutral hydrogen atoms. Cross-sections for the latter were calculated with the more generalized formula given by Steenbock \& Holweger (1984). Due to a complete lack of data, the collisional parameter $Q$ (maximum cross-section in units of $\pi a_{0}^{2}$ ) in this formula was set equal to the value of the respective electron collision (derived via the different approximations for optically allowed and forbidden bound-bound and bound-free collisions described above) and was additionally scaled with an empirical factor $S_{\mathrm{H}}=0.1$ (Holweger 1996).

\section{Non-LTE calculations for the Sun}

Our non-LTE calculations for the Sun are based on the model atom described above and employ the empirical model atmosphere of Holweger \& Müller (1974).

In Figs. 2 and 3 the resulting departure coefficients $b_{i}=n_{i, \mathrm{NLTE}} / n_{i, \mathrm{LTE}}$ are shown for a calculation with the model atom for Si I and Si II, respectively. The numbers on the left of the diagrams correspond to energy level numbers specified in Table 1 . In the solar photosphere at $\tau \approx 0.1$ about $\approx 30 \%$ of the silicon atoms are neutral while $\approx 70 \%$ are singly ionized. Our calculations show that most of Si II is present in the ground state. Therefore it is not surprising that the corresponding departure coefficient indicates almost perfect LTE conditions $\left(b_{i} \approx 1\right)$ in the Sun. The same is true for the ground state of Si I. Furthermore, throughout the photosphere $\left(\log \tau_{5000} \geq-2\right)$, deviations from LTE are almost negligible for excited levels of Si I. In contrast, most excited levels of $\mathrm{Si}$ II are overpopulated with respect to LTE. In both ionization stages there are groups of energy levels whose departure coefficients closely coincide. This is due to very small energy 


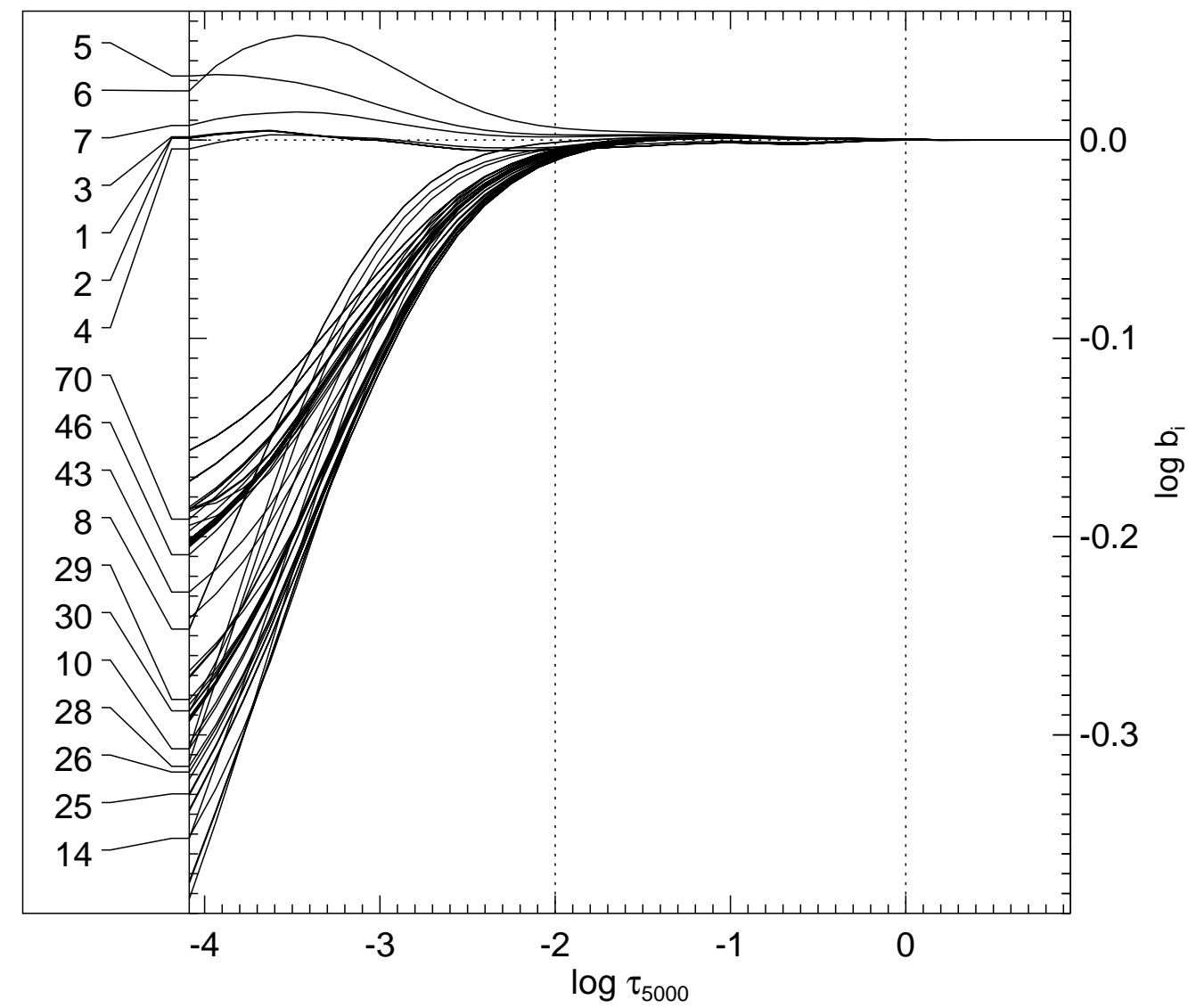

Fig. 2. Departure coefficients of Si I in the Sun.

differences within these groups and consequently a strong collisional coupling.

\subsection{Abundance analysis}

To enable a direct comparision between former LTE abundance determinations and the present work, the line list (Table 3) is essentially that adopted by Holweger (1973), except for the lines for which no departure coefficients were available from the non-LTE calculations. The present sample consists of 18 Si I lines from 10 different multiplets and two Si II lines from the same multiplet. The wavelengths and energy values $\chi_{i}$ of the lower levels of a line transition are taken from Fuhr \& Wiese (1998) as found on the NIST server. The equivalent widths of Holweger (1973) refer to the center of the solar disk. Therefore the present abundance determination was carried out for the center of the disk $(\mu=1)$ and a constant microturbulence of $\xi=1.0 \mathrm{~km} \mathrm{~s}^{-1}$ was used.

Line broadening by collisions with hydrogen atoms is treated as pure van der Waals broadening. The broadening parameter $C_{6}$ for the individual lines was calculated from the mean square atomic radii of the corresponding energy levels, based on the approximation given by Unsöld (1955). In many applications, this approximation has turned out to underestimate the real damping constants, resulting in a systematic increase of abundance with equivalent width. This was corrected by applying a correction $\Delta \log C_{6}$, either derived empirically or from quantum mechanical calculations (Steffen 1985; O'Mara 1976). However, no increase with equivalent width is present for the silicon abundances, as can be seen from Fig. 4. For this reason, no correction is necessary and a value of $\Delta \log C_{6}=0$ was adopted for this abundance determination. This agrees with Holweger (1973) and, moreover, confirms the upper limit of $\Delta \log C_{6} \approx 0.3$ given in that work. For line broadening by electron collisions, the approximations according to Griem (1968) (ions) and Cowley (1971) (neutrals) were applied. For the Si I and Si II lines used here, radiation damping is small compared to collisional broadening, and the classical approximation for $\gamma_{\mathrm{rad}}$ is used. Oscillator strengths for all lines except for $\lambda 7034(9-56)$ and $\lambda 7226(7-37)$ are tabulated in the recent compilation by Fuhr \& Wiese (1998). The derived abundances however show a large scatter with a standard deviation of \pm 0.26 dex. The mean silicon abundance is $\log \epsilon_{\mathrm{LTE}}=7.468$ (LTE) and $\log \epsilon_{\mathrm{NLTE}}=7.456$ (non-LTE), respectively (Fig. 5b). Taking the oscillator strengths of Wiese et al. (1969) leads to a somewhat lower non-LTE abundance of $\log \epsilon_{\mathrm{NLTE}}=7.405$ and a slightly smaller abundance scatter of 0.24 dex. From former investigations it is known that a much higher accuracy is possible for abundance determinations in the Sun. Obviously the internal accuracy of the Si I $f$-values in the Wiese et al./Fuhr \& Wiese compilations is rather low. Consequently, these values were not taken into 


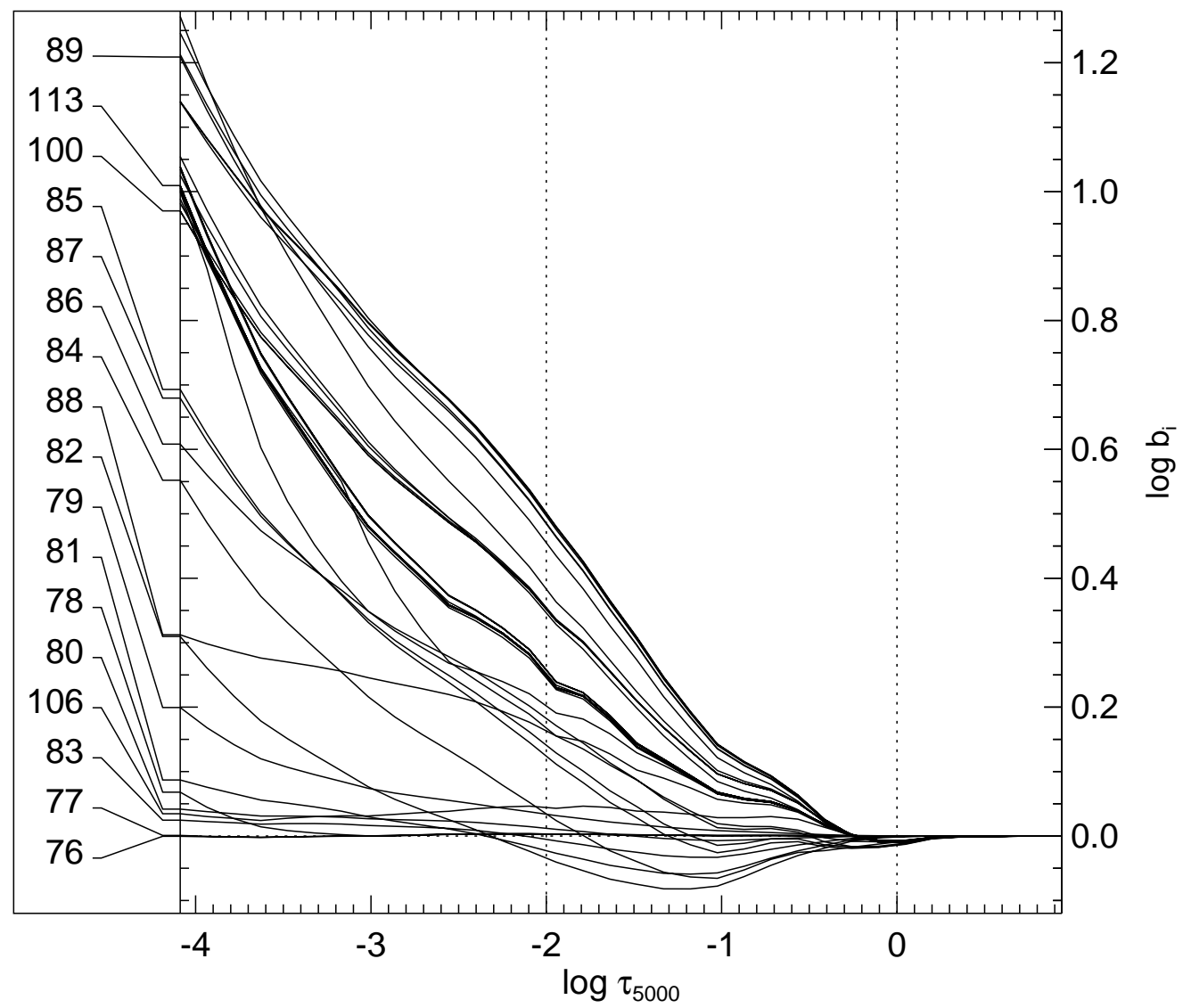

Fig. 3. Departure coefficients of Si II in the Sun.

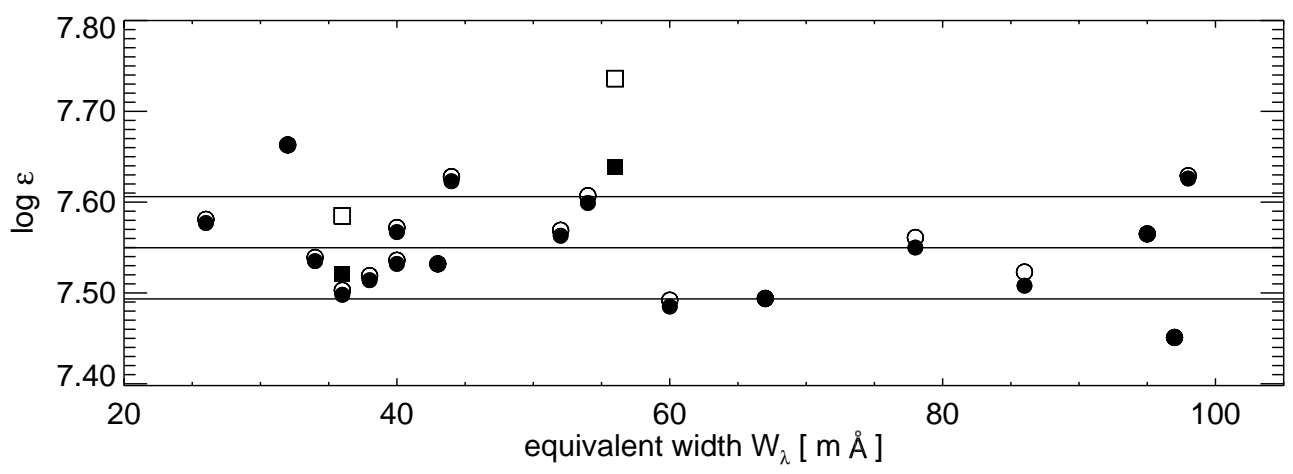

Fig. 4. Solar silicon abundance for the lines in Table 3 over equivalent width $W_{\lambda}$. Filled symbols represent non-LTE values, unfilled LTE abundances (circles for Si I, squares for Si II). The horizontal lines illustrate the weighted mean and the standard deviation (solid).

account any further. Nevertheless this set of $\log g f$-values can still be applied for the model atom. More satisfactory results were achieved with the older experimental oscillator strenghts of Garz (1973) which apparently have been adopted in the compilation by Kurucz (1993) and in the database VALD. Becker et al. (1980) have revised the absolute scale for Garz's $\log g f$-values which resulted in a general correction of +0.1 dex. Because of the small intrinsic scatter of abundances, the Garz/Becker et al. $f$-values are used for all Si I lines in this abundance analysis.

For the two Si II lines, different sources for the $\log g f$ values were found to agree within $0.07 \mathrm{dex}$. The oscillator strengths of Wiese et al. (1969)/Fuhr \& Wiese (1998) were not used, instead those derived by Froese-Fischer (1968) were choosen.

The silicon abundances determined with LINFOR from the equivalent widths of the individual lines are listed in Table 3 and illustrated in Fig. 5a.

To account for larger uncertainties in oscillator strengths quoted for some Si I lines with wavelengths $\lambda>6000 \AA$, these lines have been entered with a half weight in the final abundance. Furthermore, the strongest Si I lines with equivalent widths $W_{\lambda}>90 \mathrm{~m} \AA$ were found to be extremely sensitive to collisional line broadening 

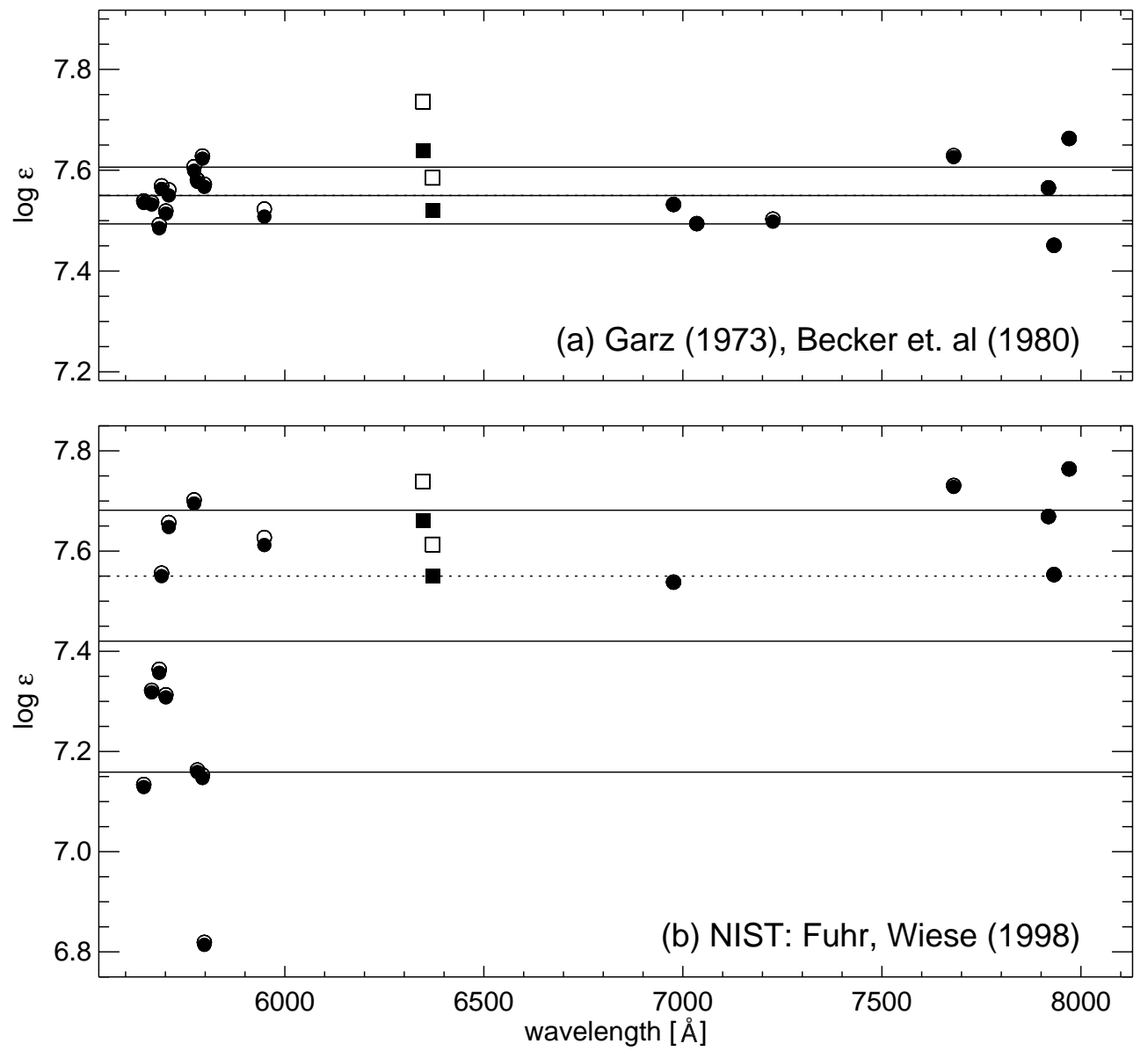

Fig. 5. Solar silicon abundance for the lines in Table 3: a) Corrected oscillator strengths by Garz (1973) and Becker et al. (1980). b) Oscillator strengths by Fuhr \& Wiese (1998). Filled symbols represent non-LTE values, unfilled LTE abundances (circles for Si I, squares for Si II). The horizontal lines illustrate the weighted mean and the standard deviation (solid) and the former (and confirmed) abundance of $\log \epsilon_{\mathrm{Si}}=7.55$ (dashed).

(van der Waals, Stark), with comparatively large uncertainties in abundance. Consequently the affected lines $\lambda \lambda 7680,7918$ and 7932 were half weighted, too. The two Si II lines are much more susceptible to uncertainties in the atomic data for the non-LTE calculations. Hence they were given half weight as well.

With the mentioned weights, an LTE abundance of $\log \epsilon_{\mathrm{LTE}}=7.560 \pm 0.066$ and a non-LTE abundance of $\log \epsilon_{\mathrm{NLTE}}=7.550 \pm 0.056$ was derived, implying a mean non-LTE correction of $\Delta \log \epsilon=-0.010$. Holweger (1973) used almost the same line list but with the original oscillator strenghts measured by Garz (1973) leading to a LTE abundance of $\log \epsilon=7.65$. With the systematic correction of the $\log g f$-values, the abundance was redetermined to $\log \epsilon=7.55$ (Becker et al. 1980). Their line sample is very similar to that used in the present work and the $f$-values were taken from the same source. There are only small differences in the atmosphere applied by Holweger (1973), mainly slightly different abundances of the main electron donors $\mathrm{Fe}, \mathrm{Mg}$ and $\mathrm{Si}$, and a depth-dependent microturbulence (see Holweger 1971). Since the present study showed that for Si II and especially Si I lines in the Sun, nonLTE effects are generally small, it is not surprising that the former silicon abundance could be reproduced almost exactly.

Several parameters have been varied to investigate their influence on the silicon abundance. Table 4 shows the deviations from the abundance derived for the above described standard model. In general, the uncertainties of atomic data for the non-LTE calculations like line broadening parameters, photoionization and collision cross-sections have only a minor effect on the resulting departure coefficients. However, uncertainties in the parameters for the abundance determination produce larger, altough still small, abundance deviations. Radiative damping has only little effect on the result. As already noticed by Holweger (1973) some Si I lines are quite sensitive to Stark broadening. If Stark broadening is completely neglected, the abundances for $\lambda \lambda 7680,7918$ and 7932 increase by 0.11 dex and by 0.08 dex for $\lambda 7034$. This effect is smaller $(<0.03$ dex $)$ for most of the remaining lines. Much stronger is the influence of the van der Waals broadening parameter $\log C_{6}$. A value of $\Delta \log C_{6}=+1.0$ would lead to a decrease in abundance by -0.056 dex. Again, the spectral lines $\lambda \lambda 7680,7918$ and 7932 are most sensitive. Adopting a microturbulence of $\xi=0.8 \mathrm{~km} \mathrm{~s}^{-1}$ instead of 
Table 3. Line list used for the abundance analysis in the Sun: wavelength, excitation potential $\chi_{i}$ of lower level, equivalent width $W_{\lambda}$ (center of disk), silicon abundances and non-LTE corrections $\Delta \log \epsilon=\log \epsilon_{\mathrm{NLTE}}-\log \epsilon_{\mathrm{LTE}}$. Oscillator strengths $\log g f$ of Si I: Garz (1973), corrected by Becker et al. (1980); Si II: Froese-Fischer (1968).

\begin{tabular}{lrccccc}
\hline \multicolumn{1}{c}{$\begin{array}{c}\lambda \\
(\AA)\end{array}$} & $\begin{array}{r}\text { mult. } \\
\text { nr. }\end{array}$ & $\begin{array}{c}\chi_{i} \\
(\mathrm{eV})\end{array}$ & $\log g f$ & $\begin{array}{c}W_{\lambda} \\
(\mathrm{m} \AA)\end{array}$ & $\log \epsilon$ & $\Delta \log \epsilon$ \\
\hline Si I: & & & & & & \\
5645.611 & 10 & 4.9296 & -2.040 & 34 & 7.535 & -0.004 \\
5665.554 & 10 & 4.9201 & -1.940 & 40 & 7.532 & -0.004 \\
5684.485 & 11 & 4.9538 & -1.550 & 60 & 7.485 & -0.007 \\
5690.427 & 10 & 4.9296 & -1.770 & 52 & 7.563 & -0.006 \\
5701.105 & 10 & 4.9296 & -1.950 & 38 & 7.514 & -0.005 \\
5708.397 & 10 & 4.9538 & -1.370 & 78 & 7.550 & -0.011 \\
5772.145 & 17 & 5.0823 & -1.650 & 54 & 7.599 & -0.008 \\
5780.384 & 9 & 4.9201 & -2.250 & 26 & 7.577 & -0.004 \\
5793.071 & 9 & 4.9296 & -1.960 & 44 & 7.623 & -0.005 \\
5797.860 & 9 & 4.9538 & -1.950 & 40 & 7.567 & -0.005 \\
5948.540 & 16 & 5.0823 & -1.130 & 86 & 7.508 & -0.015 \\
6976.520 & 60 & 5.9537 & -1.070 & 43 & 7.532 & $0.000^{a}$ \\
7034.901 & 42.10 & 5.8708 & -0.780 & 67 & 7.493 & $-0.001^{a, b}$ \\
7226.208 & 21.05 & 5.6135 & -1.410 & 36 & 7.498 & -0.005 \\
7680.265 & 36 & 5.8625 & -0.590 & 98 & 7.626 & $-0.003^{b}$ \\
7918.382 & 57 & 5.9537 & -0.510 & 95 & 7.565 & $0.000^{b}$ \\
7932.348 & 57 & 5.9639 & -0.370 & 97 & 7.451 & $0.000^{b}$ \\
7970.305 & 57 & 5.9639 & -1.370 & 32 & 7.663 & $0.000^{a}$ \\
Si II: & & & & & & \\
6347.110 & 2 & 8.1210 & 0.260 & 56 & 7.639 & $-0.097^{c}$ \\
6371.370 & 2 & 8.1210 & -0.040 & 36 & 7.521 & $-0.064^{c}$ \\
\hline
\end{tabular}

a Larger error in oscillator strength.

$b$ Strongly sensitive to collisional line broadening.

c Susceptable to uncertainties in model atom.

$\xi=1.0 \mathrm{~km} \mathrm{~s}^{-1}$ would lead to a small increase of the nonLTE abundance by +0.012 dex. Replacing the Holweger \& Müller photospheric model by an ATLAS9 model (Kurucz 1992) $\left(T_{\text {eff }}=5780 \mathrm{~K}, \log g=4.44\right)$ results in a considerably lower mean non-LTE abundance of 7.448 dex. Note that the described variations of the model parameters do not represent real error limits but rather demonstrate their different influence on the resulting abundance.

\subsection{Abundance correction due to granulation}

For the present calculations, always static, plane-parallel models are applied which cannot account for horizontal temperature inhomogeneities associated with convection. These inhomogeneities are believed to have a small but non-negligible effect on the photospheric abundances, making further corrections necessary. In a first attempt, Steffen (2000a) determined abundance corrections for various elements from $2 \mathrm{D}$ hydrodynamics simulations which yield temperature and pressure fluctuations of the solar surface. Through comparison of 1D and 2D models, both with a depth-independent microturbulence of $1.0 \mathrm{~km} \mathrm{~s}^{-1}$,
Table 4. Abundance differences due to different model assumptions for the Sun. Silicon non-LTE mean abundances, mean non-LTE corrections and deviations $(\Delta \log \epsilon)_{\mathrm{d}}$ from the result for the standard model.

\begin{tabular}{|c|c|c|c|}
\hline model assumption & $\log \epsilon$ & $\Delta \log \epsilon$ & $(\Delta \log \epsilon)_{\mathrm{d}}$ \\
\hline standard model & $7.550 \pm 0.056$ & -0.010 & \\
\hline \multicolumn{4}{|l|}{ Non-LTE calculations: } \\
\hline no Stark broadening & $7.550 \pm 0.056$ & -0.010 & 0.000 \\
\hline$\Delta \log C_{6}=-1.0$ & $7.550 \pm 0.056$ & -0.010 & 0.000 \\
\hline$\Delta \log C_{6}=+0.5$ & $7.549 \pm 0.056$ & -0.011 & -0.001 \\
\hline no line transitions & $7.565 \pm 0.065$ & +0.005 & +0.015 \\
\hline all $\gamma_{\mathrm{rad}} \times 5$ & $7.549 \pm 0.056$ & -0.011 & -0.001 \\
\hline all $\sigma_{\mathrm{PI}} \times 0.75$ & $7.549 \pm 0.056$ & -0.011 & -0.001 \\
\hline all $\sigma_{\mathrm{PI}} \times 1.25$ & $7.551 \pm 0.056$ & -0.009 & +0.001 \\
\hline all $\sigma_{\mathrm{e}} \times 0.1$ & $7.543 \pm 0.055$ & -0.017 & -0.007 \\
\hline all $\sigma_{\mathrm{e}} \times 10$ & $7.557 \pm 0.061$ & -0.003 & +0.007 \\
\hline all $\sigma_{\mathrm{H}}: S_{\mathrm{H}}=0.01$ & $7.545 \pm 0.056$ & -0.015 & -0.005 \\
\hline all $\sigma_{\mathrm{H}}: S_{\mathrm{H}}=10$ & $7.556 \pm 0.060$ & -0.004 & +0.006 \\
\hline \multicolumn{4}{|l|}{ Abundance analysis: } \\
\hline$\Delta \log \gamma_{\mathrm{rad}}=-1.0$ & $7.550 \pm 0.056$ & -0.010 & 0.000 \\
\hline$\Delta \log \gamma_{\mathrm{rad}}=+1.0$ & $7.543 \pm 0.056$ & -0.009 & -0.007 \\
\hline$\Delta \log C_{4}=-0.5$ & $7.565 \pm 0.057$ & -0.010 & +0.015 \\
\hline$\Delta \log C_{4}=+0.5$ & $7.525 \pm 0.063$ & -0.009 & -0.025 \\
\hline no Stark broadening & $7.582 \pm 0.061$ & -0.011 & +0.032 \\
\hline$\Delta \log C_{6}=0.6$ & $7.519 \pm 0.061$ & -0.009 & -0.031 \\
\hline$\Delta \log C_{6}=1.0$ & $7.493 \pm 0.071$ & -0.008 & -0.056 \\
\hline$\xi=0.8 \mathrm{~km} \mathrm{~s}^{-1}$ & $7.562 \pm 0.057$ & -0.010 & +0.012 \\
\hline ATLAS & $7.448 \pm 0.058$ & -0.008 & -0.102 \\
\hline
\end{tabular}

the effect of temperature inhomogenities on the abundance can be determined. A first application is reported in Aellig et al. (1999). A more detailed description will be given in Steffen \& Holweger (2001).

For the case of silicon, M. Steffen (2000b) kindly provided granulation abundance corrections for 10 representative line transistions which permitted an interpolation for the remaining lines in Table 3. The resulting abundance corrections depend on the excitation potential $\chi_{i}$ and equivalent width. The mean granulation abundance correction for Si I line transitions with $\chi_{i} \approx 5 \mathrm{eV}$ is +0.023 and increases to 0.029 dex for $\chi_{i} \approx 6 \mathrm{eV}$. The abundance corrections for the two Si II lines are -0.014 . Considering the same weights as in the abundance determination above, a total correction of +0.021 due to granulation results. Finally, the photospheric silicon abundance becomes $\log \epsilon_{\mathrm{GC}}=7.571$, including granulation effects. In $3 \mathrm{D}$ simulations, temperature fluctuations are in general smaller than in 2D. Therefore the granulation abundance correction described here should be considered as an upper limit. 


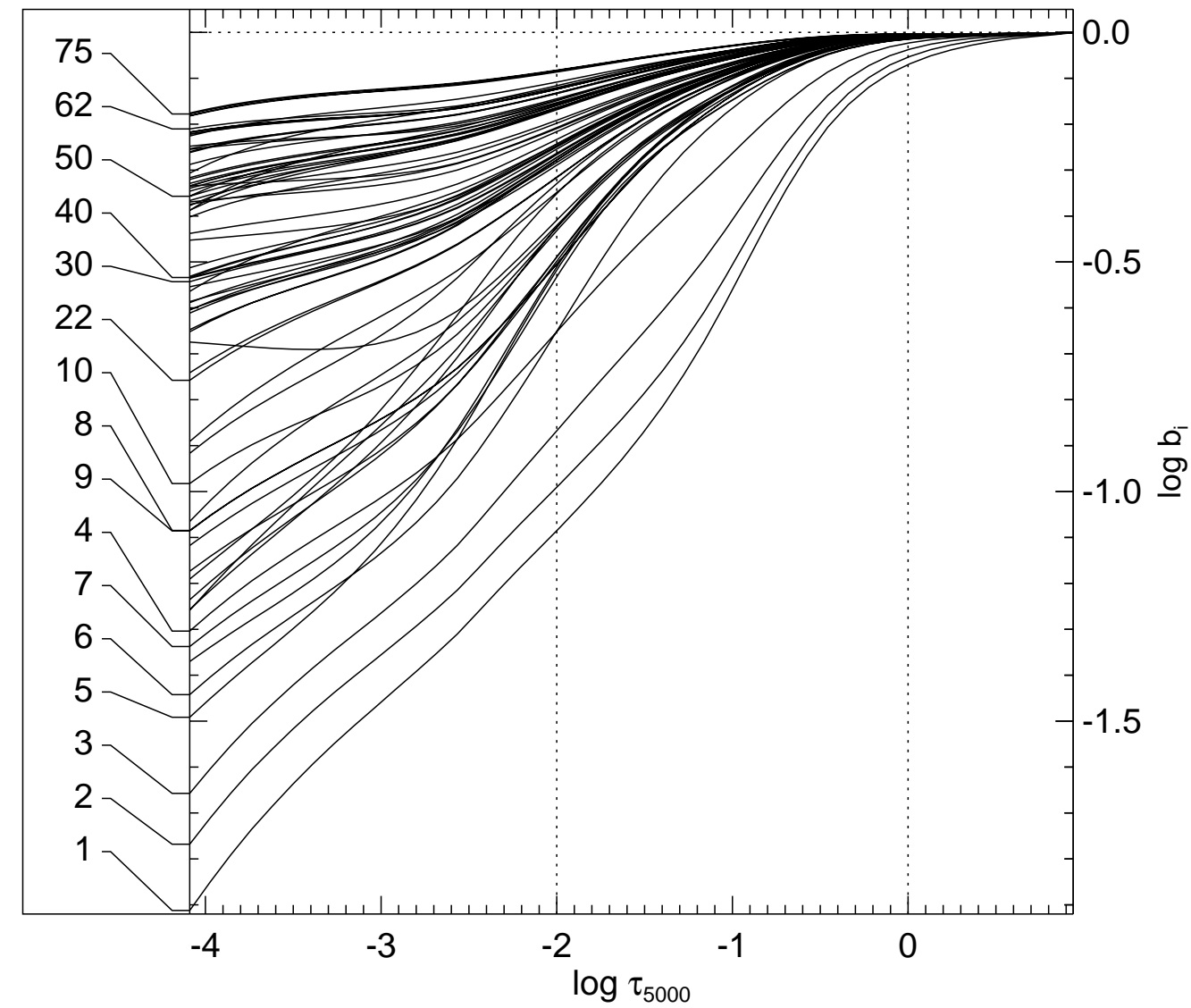

Fig. 6. Departure coefficients of Si I in Vega.

\section{Non-LTE calculations for Vega}

The Vega model atmosphere is the same as used in the non-LTE abundance analysis of nitrogen (RentzschHolm 1996). It was generated with the ATLAS9 code (Kurucz 1992), adopting $T_{\text {eff }}=9500 \mathrm{~K}, \log g=3.90$, $[\mathrm{M} / \mathrm{H}]=-0.5$, and a depth-independent microturbulence $\xi=2.0 \mathrm{kms}^{-1}$. The adopted subsolar metallicity is in accordance with recent non-LTE analyses (see Rentzsch-Holm 1996), e.g. $[\mathrm{C} / \mathrm{H}]=-0.30 \pm 0.18$ for carbon (Stürenburg \& Holweger 1991). The applied model atom is the same as for the Sun.

The resulting departure coefficients of Si I (Fig. 6) show that low-lying energy levels are strongly underpopulated with respect to LTE, implying substantial overionisation. Most of the silicon is in the singly ionized stage. The two lowest energy levels of Si II are almost perfectly in LTE (Fig. 7) but most of the excited Si II levels are overpopulated with respect to LTE. The ground state of Si III is also illustrated in Fig. 7. Again, strong overpopulation is obvious. However, the fraction of silicon present as Si III is still small compared to Si II.

\subsection{Abundance analysis}

The abundance analysis of Vega is based on low-noise high-resolution photographic spectra kindly provided by
Table 5. Si II line list used for the abundance analysis in Vega: Wavelength, excitation potential $\chi_{i}$ of lower level, equivalent width $W_{\lambda}$, silicon abundances and non-LTE corrections $\Delta \log \epsilon=\log \epsilon_{\mathrm{NLTE}}-\log \epsilon_{\mathrm{LTE}}$. Oscillator strengths: VALD.

\begin{tabular}{ccccccc}
\hline $\begin{array}{c}\lambda \\
(\AA)\end{array}$ & $\begin{array}{c}\text { mult. } \\
\mathrm{nr} .\end{array}$ & $\begin{array}{c}\chi_{i} \\
(\mathrm{eV})\end{array}$ & $\log g f$ & $\begin{array}{c}W_{\lambda} \\
(\mathrm{m} \AA)\end{array}$ & $\log \epsilon$ & $\Delta \log \epsilon$ \\
\hline 3862.595 & 1 & 6.8575 & -0.817 & 89 & 6.980 & +0.050 \\
4128.054 & 3 & 9.8367 & 0.316 & 67 & 6.911 & -0.093 \\
4130.872 & 3 & 9.8388 & -0.824 & 84 & 6.928 & $-0.106^{a}$ \\
4130.894 & 3 & 9.8388 & 0.476 & $a$ & 6.928 & $-0.106^{a}$ \\
5041.024 & 5 & 10.0664 & 0.291 & 43 & 6.980 & $-0.062^{b}$ \\
5055.984 & 5 & 10.0739 & 0.593 & 77 & 6.970 & $-0.062^{c}$ \\
5056.317 & 5 & 10.0739 & -0.359 & $c$ & 6.970 & $-0.062^{c}$ \\
\hline
\end{tabular}

$a, c \quad$ Si II blend, combined equivalent width.

$b \quad$ Blend with Fe I.

R. Griffin (Griffin \& Griffin 1977) covering the visible spectral region. Seven Si II lines (Table 5) were found to be suitable for a reliable abundance determination, whereas all Si I lines are much too weak. Equivalent widths were measured directly from the tracings. Line broadening is treated in the same way as in the foregoing solar abundance determination. As for the Sun, the oscillator strengths compiled by Wiese et al. (1969) and 


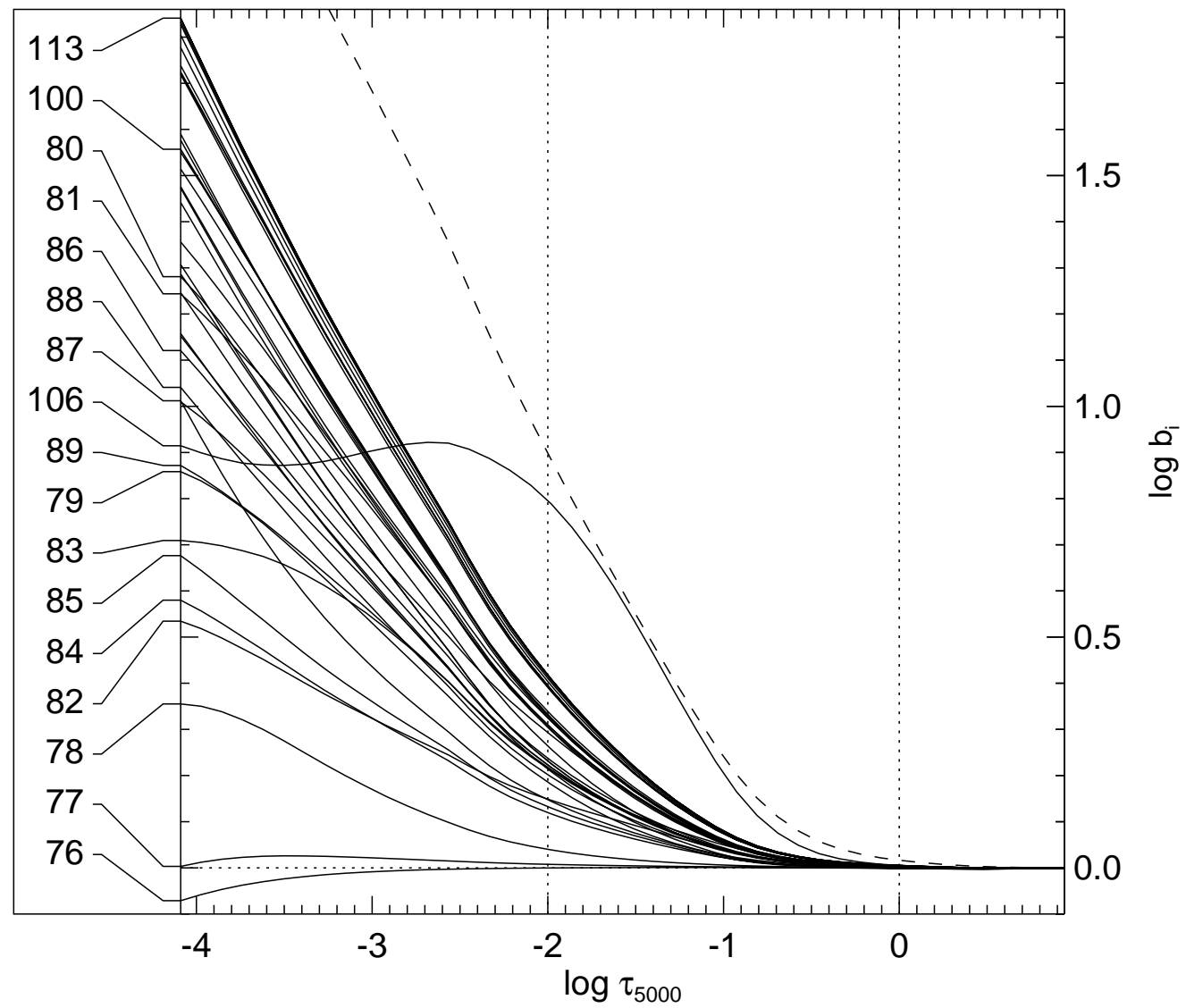

Fig. 7. Departure coefficients of Si II in Vega. The dashed line represents the ground state of Si III.

Fuhr \& Wiese (1998) produce a larger abundance scatter $(\sigma=0.094 \mathrm{dex})$ than the values taken from VALD $(\sigma=0.029 \mathrm{dex})$. For this reason the latter set is used. The lines marked with ${ }^{a}$ and ${ }^{c}$ in Table 5 are close blends of Si II lines. Consequently, the listed equivalent widths refer to the entire blend, and these blends are only considered with half weight. The line marked with ${ }^{b}$ is also weighted half because it is blended with a Fe I line.

The non-LTE corrections are all negative with values between -0.062 and -0.106 with the exception of $\lambda 3862$. For this particular line the non-LTE correction is positive $(+0.05)$ because the departure coefficient of the upper level exceeds that of the lower level, contrary to the other lines.

From these 7 lines a weigthed LTE abundance of $\log \epsilon_{\mathrm{LTE}}=7.005$ was derived. The small mean non-LTE correction of $\Delta \log \epsilon=-0.054$ finally leads to a silicon abundance of $\log \epsilon_{\mathrm{NLTE}}=6.951$ with a standard deviation of 0.029 dex. The estimated error limits, including uncertainties in the equivalent widths, are $\approx 0.1$ dex. Table 6 shows the influence of the line broadening parameters and the microturbulence on the mean non-LTE abundance compared to the described standard Vega model. For a smaller microturbulence of $\xi=1.5 \mathrm{~km} \mathrm{~s}^{-1}$, the resulting non-LTE abundances increases by +0.228 dex. Unlike the Sun, van der Waals broadening is less important than Stark broadening, as expected for an A star. Completely
Table 6. Abundance differences due to different model assumptions for the abundance determination of Vega. Silicon non-LTE mean abundances, mean non-LTE corrections and deviations $(\Delta \log \epsilon)_{\mathrm{d}}$ from the result for the standard model.

\begin{tabular}{lccr}
\hline model assumption & $\log \epsilon$ & $\Delta \log \epsilon$ & $(\Delta \log \epsilon)_{\mathrm{d}}$ \\
\hline standard model & $6.951 \pm 0.029$ & -0.054 & \\
\hline$\Delta \log \gamma_{\mathrm{rad}}=-1.0$ & $6.952 \pm 0.029$ & -0.054 & 0.001 \\
$\Delta \log \gamma_{\mathrm{rad}}=+1.0$ & $6.935 \pm 0.028$ & -0.054 & -0.016 \\
$\Delta \log C_{4}=-0.5$ & $7.014 \pm 0.017$ & -0.062 & +0.063 \\
$\Delta \log C_{4}=+0.5$ & $6.861 \pm 0.052$ & -0.044 & -0.090 \\
no Stark broadening & $7.096 \pm 0.047$ & -0.083 & +0.145 \\
$\Delta \log C_{6}=1.5$ & $6.951 \pm 0.029$ & -0.053 & 0.000 \\
$\xi=1.5 \mathrm{~km} \mathrm{~s}^{-1}$ & $7.179 \pm 0.077$ & -0.089 & +0.228 \\
\hline
\end{tabular}

neglecting the Stark broadening causes an abundance deviation of +0.119 dex from the standard model.

For comparison, Hill (1995) and Lemke (1990) obtained values of $\log \epsilon_{\mathrm{Si}}=6.86$ and $\log \epsilon_{\mathrm{Si}}=6.94$, respectively, which is in good agreement with the result of this work.

The abundance of silicon in Vega differs by -0.599 dex from the solar value, confirming the deficiency of Si found by other authors. 


\section{Conclusions}

Non-LTE effects of silicon in the Sun were found to be small. The solar silicon abundance becomes $\log \epsilon_{\mathrm{NLTE}}=7.550 \pm 0.056$ with a mean non-LTE correction of $\Delta \log \epsilon=-0.010$. This matches almost exactly the previous values (e.g. Becker et al. 1980; Holweger 1979).

The effect of horizontal temperature inhomogeneities associated with convection on the photospheric abundance of Si has also been considered. According to preliminary results by Steffen (2000b), based on 2D hydrodynamics simulations, a mean granulation abundance correction of +0.021 dex is probably a safe upper limit, leading to a silicon abundance of 7.571 .

As a by-product of the solar analysis, an assessment of the accuracy of the $f$-values was possible. The internal accuracy of the $f$-values of Garz (1973) is superior to those compiled by Wiese et al. (1969) and Fuhr \& Wiese (1998).

For Vega, a Si abundance of $\log \epsilon_{\mathrm{NLTE}}=6.951 \pm 0.100$ and a non-LTE correction of only $\Delta \log \epsilon=-0.054$ was derived. This confirms the value $\log \epsilon_{\mathrm{Si}}=6.94$ quoted by Lemke (1990). With respect to the Sun, an underabundance of -0.599 dex results, confirming the general metal deficiency of Vega.

Acknowledgements. The author wishes to thank H. Holweger for suggesting and supporting this work. Further thanks are due to I. Kamp and M. Hempel for their useful comments and help with non-LTE calculations and abundance analysis, M. Steffen for providing unpublished data for granulation abundance corrections and to the referee Y. Takeda for helpful comments.

\section{References}

Aellig, M. R., Holweger, H., Bochsler, P., et al. 1999, AIP Conf. Proc. 471, Solar Wind Nine, 255

Allen, C. W. 1973, Astrophysical quantities, 3rd ed. (Athlone Press, London)

Anders, E., \& Grevesse, N. 1989, GeCoA, 53, 197

Auer, L. H., \& Heasley, J. N. 1976, ApJ, 205, 165

Baschek, B., Holweger, H., \& Traving, G. 1966, Abhandlungen aus der Hamburger Sternwarte, 8, 26

Becker, U., Zimmermann, P., \& Holweger, H. 1980, GeCoA, 44,2145

Cowley, C. R. 1971, The Observ., 91, 139

Drawin, H. W. 1967, Collision and Transport Cross-Sections, Association Euratom-C.E.A., EUR-CEA-FC-383

Froese-Fischer, C. 1968, ApJ, 151, 759

Fuhr, J. R., \& Wiese, W. L. 1998, Atomic Transition Probabilities, CRC Handbook of Chemistry and Physics, 79th ed. (CRC Press), Inc.
Garz, T. 1973, A\&A, 26, 471

Gigas, D. 1988, A\&A, 192, 264

Grevesse, N., \& Sauval, A. J. 1998, Stand. Solar Comp., Space Sci. Rev., 85, 161

Griem, H. R. 1968, Phys. Rev., 165, 258

Griffin, R., \& Griffin, R. E. M. 1977, Spectroscopic Atlas of Vega, unpublished

Hill, G. M. 1995, A\&A, 294, 536

Holweger, H. 1971, A\&A, 10, 128

Holweger, H. 1973, A\&A, 26, 275

Holweger, H., \& Müller, E. A. 1974, Solar Phys., 39, 19

Holweger, H. 1979, Abundances of the elements in the sun Introductory report, The Elements and their Isotopes in the Universe, 117

Holweger, H., \& Rentzsch-Holm, I. 1995, A\&A, 303, 819

Holweger, H. 1996, Phys. Scr. T, 65, 151

Kupka, F., Piskunov, N. E., Ryabchikova, T. A., Stempels, H. C., \& Weiss W. W. 1999, A\&AS, 138, 119

Kurucz, R. L. 1992, Rev. Mex. Astron. Astrofis., 23, 181

Kurucz, R. L. 1993, SAO KURUCZ CD-ROM, Nos. 13 and 18

Lambert, D. L., \& Luck, R. E. 1978, MNRAS, 183, 79

Lemke, M., \& Holweger, H. 1987, A\&A, 173, 375

Lemke, M. 1990, A\&A, 240, 331

Martin, W. C., \& Zalubas, R. 1983, J. Phys. Chem. Ref. Data, 12,323

Omara, B. J. 1976, MNRAS, 177, 551

Piskunov, N. E., Kupka, F., Ryabchikova, T. A., Weiss, W. W., \& Jeffery, C. S. 1995, A\&AS, 112, 525

van Regemorter, H. 1962, ApJ, 136, 906

Rentzsch-Holm, I. 1996, A\&A, 305, 275

Seaton, M. J., Zeippen, C. J., Tully, J. A., et al. 1992, Rev. Mex. Astron. Astrofis., 23, 19

Seaton, M. J., Yan, Y., Mihalas, D., \& Pradhan, A. K. 1994, MNRAS, 266, 805

Sobelman, I. I., Vainshtein, L. A., \& Yukov, E. A. 1981, Excitation of atoms and broadening of spectral lines, Springer Series in Chemical Physics 7, Berlin

Steenbock, W., \& Holweger, H. 1984, A\&A, 130, 319

Steenbock, W. 1985, in Cool Stars with Excesses of Heavy Elements, ed. M. Jaschke, \& P. C. Keenan (Reidel, Dordrecht), 231

Stürenburg, S., \& Holweger, H. 1990, A\&A, 237, 125

Stürenburg, S., \& Holweger, H. 1991, A\&A, 246, 644

Steffen, M. 1985, A\&AS, 59, 403

Steffen, M. 2000a, Pacific Rim Conference on Stellar Astrophysics, Hong Kong (1999)

Steffen, M. 2000b, priv. comm.

Steffen, M., \& Holweger, H. 2001, in preparation

Stürenburg, S. 1993, A\&A, 277, 139

Takeda, Y. 1992, PASJ, 44, 309

Venn, K. A., \& Lambert, D. L. 1990, ApJ, 363, 234

Unsöld, A. 1955, Physik der Sternatmosphären (Springer, Berlin)

Wiese, W. L., Smith, M. W., \& Miles, B. M. 1969, Atomic transition probabilities., Natl. Stand. Ref. Data Ser., NSRDSNBS 22, vol. 2, Washington, DC 